\title{
Margulis Distributions for Anosov Flows
}

\author{
Mark Pollicott \\ University of Edinburgh, Department of Mathematics, James Clerk Maxwell Building, \\ King's Buildings, Mayfield Road, Edinburgh, EH9 3JZ, United Kingdom
}

\begin{abstract}
For the strong unstable foliation (or horocycle foliation) of an Anosov flow there exists a unique transverse measure called the Margulis measure. In this paper we extend Margulis' results to more general "transverse distributions" for the foliation. As an application we derive our main result: The non-zero analytic extension to a strip of the Selberg zeta function for compact surfaces of constant negative curvature persists under small perturbations in the metric. There is an equivalent formulation in terms of the Fourier transform of the correlation function.
\end{abstract}

\section{Introduction}

In the study of Axiom $A$ flows there are two functions (of a single complex variable) which contain a considerable amount of information on the dynamics of the flow the zeta function and the Fourier transform of the correlation function (which describes the way in which the flow mixes). Their meromorphic domains influence the asymptotic growth of closed orbit periods (through the zeta function [14]) and the rate of mixing of the flow (through the correlation function $[16,21]$ ).

David Ruelle has gone some way in analysing the residues which occur for the Fourier transform and he has given them some interpretation in terms of "Gibbs distributions" [22] (similar in nature to distributions in the Schwartzian sense, but which are complex valued). However, this does not broach the problem of locating the poles. There is a simple correspondence between poles for the zeta function and poles for the Fourier transform of the correlation function (within appropriate domains) which reduces this to a single problem [16].

In this article we give an approach to determining the location of the poles and to giving them, along with the residues, a clearer interpretation, at least for Anosov flows.

We shall formulate most of our results at the level of three-dimensional Anosov flows. This will be most appropriate for our main application.

Our approach to these problems is to consider transverse distributions (generalising transverse measures) for the natural foliation associated with the 
strong stable manifolds of Anosov flows. This approach is motivated by Margulis' very elegant work on transverse measures for horocycle foliations [25], although the techniques we use are very different. (We should remark that Margulis apparently used these measures in obtaining asymptotic estimates for closed geodesic lengths, demonstrating how closely related these concepts are, cf. also [14].)

Bowen profoundly influenced the study of both Axiom $A$ diffeomorphisms and flows with the use of symbolic dynamics $[4,5]$ (Bowen was obviously inspired by the Russian school $[18,19])$. This allowed many problems to be reformulated for subshifts of finite type (in the discrete case) or suspended flows (in the continuous case). For subshifts of finite type a number of powerful results can be proved using techniques originating in statistical mechanics. In particular, the Ruelle operator proves to be a very useful tool [5]. For flows the situation is slightly more involved (not least because of the difficulty in directly applying ideas from statistical mechanics). The Ruelle operator can be used again, although for flows it plays a rather more subtle role [17].

In Sect. 1 we review the basic definitions and ideas connected with Axiom $A$ flows and strong stable (horocycle) foliations. In Sect. 2 we relate this to our working format of suspended flows over subshifts (of finite type) by the use of symbolic dynamics.

In Sect. 3 we give an alternative construction of Margulis (transverse) measures, the unique transverse measure associated with the strong stable foliation. This makes use of the spectrum of (the dual to) the Ruelle operator.

In Sect. 4 we recall some results on the Fourier transform of the correlation function (which describes the rate at which the flow mixes). This is intimately connected with the spectrum of the Ruelle operator, which allows us to relate the existence of poles to the existence of certain "Margulis distributions" generalising Margulis measures.

In Sect. 5 we relate the existence of poles (where the correlation is with respect to the measure of maximal entropy) to the induced action of $\phi_{t}$ on an appropriate Banach space (subject to an orientation condition on $\phi_{t}$ ). The intermediate step is the connection with the Margulis distributions.

The Sect. 6 contains the first version of our main result: For metrics close to those of constant negative (sectional) curvature the Fourier transform of the correlation function for the geodesic flow (with the measure of maximal entropy) has an analytic extension to a strip.

In Sect. 7 we give the equivalent formulation of our results in terms of the (dynamical) zeta function. Our main result now becomes: For metrics close to those of constant negative curvature the zeta function for the geodesic flow has an analytic extension to a strip.

\section{Axiom $A$ Flows and Strong Stable Foliations}

We begin by introducing our basic object of study, a $C^{\infty}$ Axiom $A$ flow on a compact Riemannian manifold, and recall some of the basic definitions and properties. 
Let $M$ be a compact $C^{\infty}$ Riemannian manifold, and let $\phi_{t}: M \rightarrow M$ be a $C^{1+\varepsilon}$-flow. We define the nonwandering set

$$
\Omega=\left\{x \in M \mid \forall \text { open neighbourhoods } U \ni x, \exists t_{0}>0 \text { s.t. } U \cap\left(\bigcup_{t>t_{0}} \phi_{t} U\right) \neq \phi\right\} .
$$

A flow satisfies Axiom $A$ if the non-wandering set is the disjoint union of a finite number of hyperbolic fixed points and a finite number of $\phi_{t}$ invariant sets $A$ satisfying:

1) $\left.\phi\right|_{A}$ has no fixed points and closed orbits of $\left.\phi\right|_{A}$ are dense in $\Lambda$.

2) $\left.\phi\right|_{A}$ is transitive (i.e. $A$ contains a dense orbit).

3) $T_{A} M$ splits into $3 D \phi_{t}$-invariant continuous sub-bundles $T_{A} M=E \oplus E^{u} \oplus E^{s}$ with $E$ tangent to the flow direction and

$$
\begin{aligned}
& \left\|D \phi_{t}(v)\right\| \leqq C e^{-\lambda t}\|v\| \text { for } v \in E^{s}, \quad t \geqq 0, \\
& \left\|D \phi_{-t}(v)\right\| \leqq C e^{-\lambda t}\|v\| \text { for } v \in E^{u}, \quad t \geqq 0 .
\end{aligned}
$$

(Each such $\Lambda$ is called a basic set.) [27]

We call the flow $\phi_{t}: \Lambda \rightarrow \Lambda$ (topologically) weak-mixing if there are no nontrivial solutions to $F_{\phi_{t}}=e^{i a t} F$ for $F \in C(\Lambda)$ and $a>0$. Not weakmixing flows are topologically conjugate to constant suspensions of Axiom $A$ diffeomorphisms and consequently much of their analysis has already been performed at the level of diffeomorphisms [5]. Thus we shall be interested generally only in weak-mixing Axiom $A$ flows.

We call $\phi_{t}$ Anosov if we can replace $\Lambda$ by $M$ in condition 3), i.e. the entire manifold is hyperbolic.

We call an Anosov flow transitive if $\exists x \in M$ with a dense orbit.

Having established the definitions for the flow we shall now consider the associated ergodic theory.

For any $\phi_{t}$-invariant measure $\varrho$ we define the entropy $h(\varrho)$ of $\phi$ (with respect to $\varrho)$ to be the entropy of the time-one homeomorphism $\phi_{1}: \Lambda \rightarrow \Lambda$. There are an infinite number of (ergodic) invariant measures and their entropies are bounded above. We define the (topological) entropy by

$$
h(\phi)=\sup \{h(\varrho) \mid \varrho \text { is } \phi \text {-invariant }\} \text {. }
$$

The entropy has a number of different definitions and gives a quantitative estimate on the "randomness" of the flow. For Axiom $A$ flows the entropy is always strictly positive and there is a unique $\phi$-invariant measure $m$ where the supremum is attained [i.e. $h(m)=h(\phi)]$. This measure $m$ is called the maximal measure (or Bowen measure, or measure of maximal entropy) [7].

We now consider some slightly more geometric aspects of the flow. The hyperbolicity condition on the basic set is defined in terms of the tangent bundle $T_{\Lambda} M$ but has a direct influence on the manifold $M$. For $x \in A$ we can define the strong unstable and strong stable manifolds as

$$
\begin{aligned}
& W^{\mathrm{su}}(x)=\left\{y \in M \mid d\left(\phi_{-t} x, \phi_{-t} y\right) \rightarrow 0 \text { as } t \rightarrow+\infty\right\}, \\
& W^{\mathrm{ss}}(x)=\left\{y \in M \mid d\left(\phi_{t} x, \phi_{t} y\right) \rightarrow 0 \text { as } t \rightarrow+\infty\right\}
\end{aligned}
$$


respectively. [We shall also sometimes use $W_{\varepsilon}^{\text {ss }}(x)=\left\{y \in M \mid d\left(\phi_{t} x, \phi_{t} y\right) \leqq \varepsilon, \forall t \geqq 0\right\}$ which forms a neighbourhood of $x$ in $W^{\text {ss }}(x)$ for $\varepsilon>0$.]

In accordance with their names $W^{\text {su }}(x)$ and $W^{\text {ss }}(x)$ form $C^{\infty}$-immersed submanifolds of dimensions $n=\operatorname{dim} E^{u}$ and $m=\operatorname{dim} E^{s}$ respectively. Furthermore, for $x \in \Lambda$ we have $T_{x} W^{\mathrm{su}}(x)=E_{x}^{\mathrm{u}}$ and $T_{x} W^{\mathrm{ss}}(X)=E_{x}^{\mathrm{s}}$ (cf. [2]).

For Anosov flows the strong-stable manifolds form a foliation for $M$ in the usual sense. However, although each leaf is $C^{\infty}$ in general the foliation itself is only Holder continuous.

We shall denote this foliation by $F^{\text {ss }}$ (cf. [8]).

We should also consider the effect of the flow $\phi_{t}: \Lambda \rightarrow \Lambda$ on these foliations. The main point is that for $x \in \Lambda$ we have $\phi_{t} W^{\text {ss }}(x)=W^{\text {ss }}\left(\phi_{t} x\right)$ and $\phi_{t} W^{\text {su }}(x)=W^{\text {su }}\left(\phi_{t} x\right)$, i.e. the flow preserves the foliation by taking leaves to leaves (without necessarily preserving the individual leaves).

We can use these foliations to generate new foliations $F^{\text {wu }}$ and $F^{\text {ws }}$ whose leaves are the $(n+1)$-dimensional weak unstable manifolds and $(m+1)$-dimensional weak stable manifolds given by

$$
W^{\mathrm{wu}}(x)=\bigcup_{t \in \mathbb{R}} W^{\mathrm{su}}\left(\phi_{t} x\right) \quad \text { and } \quad W^{\mathrm{ws}}(x)=\bigcup_{t \in \mathbb{R}} W^{\mathrm{ss}}\left(\phi_{t} x\right),
$$

respectively $(x \in \Lambda)$. For these foliations the individual leaves are now invariant under the flow. (The weak unstable manifolds form transverse sections to the foliation $F^{\mathrm{ss}}$.)

An important example is the geodesic flow on the (three-dimensional) unit tangent bundle of a compact surface of strictly negative curvature. This is a transitive Anosov flow for which the leaves of $F^{\text {ss }}$ are one dimensional and the leaves of $F^{\text {wu }}$ are two dimensional.

For geodesic flows all of these foliations are known to be $C^{1}$ (and so in particular Holder continuous with exponent one) [12].

\section{The Symbolic Dynamics}

To study problems about Axiom $A$ flows it is frequently convenient to reduce them to questions at the level of the simpler symbolic or suspended flows. This approach has been developed by such people as Sinai, Ratner, Bowen, and Ruelle. We shall now give a brief account, explaining only those parts of the theory relevant to subsequent sections (for full details, cf. [4]).

If $\phi_{t}: M \rightarrow M$ is our Axiom $A$ flow then we construct $(n+m)$-dimensional differentiable, disjoint closed sections $T_{1}, \ldots, T_{k}$ transverse to the flow $\phi \mid \Lambda$. [They can be assumed to lie in the intersection of the interiors of $(n+m)$-dimensional $C^{\infty}$ closed discs $D_{i}$ transverse to the flow and $\Lambda$.] Furthermore, these sections can be chosen arbitrarily small (Diam $T_{i}<\varepsilon$, say) and assumed to capture all of the flow in the sense that for any $x \in A$ we can choose sequences $t_{p} \uparrow+\infty$ and $t_{-q} \downarrow-\infty$ (for $p, q \in \mathbb{Z}^{+}$) such that $\left|t_{i}-t_{i+1}\right|<\varepsilon$ and $\phi_{t_{p}} x, \phi_{t_{-q}} x \in T_{1} \cup \ldots \cup T_{k}$. With respect to the topology on $\bigcup_{i} D_{i} \cap \Lambda$ we can consider $\partial T_{i}$ and int $T_{i}, i=1, \ldots, k$. Let $x \in \Lambda$ be a point whose orbit under $\phi_{t}$ intersects the $T_{i} s$ in their interiors in the sequence $\ldots T_{x_{-1}}, T_{x_{0}}, T_{x_{1}}, \ldots$, then $x$ gives rise to a sequence $x=\left(x_{n}\right)_{n=-\infty}^{+\infty} \in \prod_{n=-\infty}^{+\infty}\{1, \ldots, k\}$. 
The sequence $x$ records the orbit of the point $x$ (and the zeroth term $x_{0} \in\{1, \ldots, k\}$ determines the last section traversed by the orbit whilst $x$ is flowing forwards in time). The hyperbolicity of the flow on $\Lambda$ permits these sections $\left\{T_{i}\right\}$ to be chosen so that they give very precise information on the corresponding orbit. (In particular, no two distinct orbits in $A$ give rise to the same sequence.) Let $P: \bigcup_{i} T_{i} \rightarrow \bigcup_{i} T_{i}$ be the Poincaré map given by $P(x)=\phi_{\alpha}(x)$, where $\alpha=\inf \left\{t>0 \mid \phi_{t} x \in \bigcup_{i} T_{i}\right\}$. We can introduce a $k \times k$ aperiodic matrix with entries either one or zero by

$$
A(i, j)= \begin{cases}1 & \text { if } P\left(\operatorname{int} T_{i}\right) \cap\left(\operatorname{int} T_{j}\right) \neq \phi \\ 0 & \text { otherwise }\end{cases}
$$

We define a space of sequences $\Sigma_{A}$ by

$$
\Sigma_{A}=\left\{\underline{x} \in \prod_{-\infty}^{+\infty}\{1, \ldots, k\} \mid A\left(x_{n}, x_{n+1}\right)=1, n \in \mathbb{Z}\right\} .
$$

Without loss of generality we can assume that the matrix $A$ is aperiodic.

We have a topology on $\Sigma_{A}$ induced from the product of the discrete topology on $\{1, \ldots, k\}$ and with respect to this topology $\Sigma_{A}$ is a compact zero-dimensional space. We define a metric for this topology by

$$
d(\underline{x}, \underline{y})=\sum_{n=-\infty}^{+\infty} \frac{e\left(x_{n}, y_{n}\right)}{2^{|n|}}, \text { where } e(\alpha, \beta)=\begin{array}{ll}
0 & \text { if } \alpha=\beta \\
1 & \text { otherwise. }
\end{array}
$$

The shift $\sigma: \Sigma_{A} \rightarrow \Sigma_{A}$ is defined by $(\sigma x)_{n}=x_{n+1}$, and with respect to the above topology is a homeomorphism.

The sections $\left\{T_{i}\right\}_{i=1}^{k}$ can be chosen so that the map $\pi: \Sigma_{A} \rightarrow \bigcup_{i=1}^{k} T_{i}$ with

$$
\pi(\underline{x})=\bigcap_{n=-\infty}^{+\infty} \overline{P^{-n}\left(\operatorname{int} T_{x_{n}}\right)}
$$

is well-defined. Define $r: \Sigma_{A} \rightarrow \mathbb{R}$ by

$$
r(\underline{x})=\inf \left\{t>0 \mid \phi_{t}(\pi(\underline{x})) \in T_{x_{1}}\right\},
$$

then $r$ is strictly positive (and uniformly bounded away from zero).

The sections $T_{i}$ can be chosen so that $r(\underline{x})=r(\underline{y})$ whenever $x_{n}=y_{n}$ for $n \geqq 0$. (Strictly speaking, we can no longer think of $D_{i}$ as being $C^{\infty}$ cf. [19] for details.)

We define

$$
\Sigma_{A}^{r}=\left\{(\underline{x}, t) \in \Sigma_{A} \times \mathbb{R} \mid 0 \leqq t \leqq r(x)\right\},
$$

where $(x, r(x))$ and $(\sigma x, 0)$ are identified. This space inherits a product topology from $\Sigma_{A}$ and $\mathbb{R}$.

We define the suspended flow $\sigma_{t}^{r}: \Sigma_{A}^{r} \rightarrow \Sigma_{A}^{r}$ locally by $\sigma_{t}^{r}(\underline{x}, s)=(\underline{x}, s+t)$, taking account of the identifications where appropriate. We can extend the map $\pi$ to $\pi: \Sigma_{A}^{r} \rightarrow \Lambda$ by $\pi(x, \hat{t})=\phi_{1} \pi(x)$.

The suspended flow $\sigma^{r}$ gives a very accurate model of the original Axiom $A$ flow $\phi_{t}: M \rightarrow M$. We summarise some of the main results in the following proposition: 
Proposition 1 (Bowen) [4]. There exists a choice of $\left\{T_{i}\right\}$ such that

(i) The map $\pi: \Sigma_{A}^{r} \rightarrow \Lambda$ is Hölder, surjective, bounded-one and one-one on a residual set.

(ii) The map $\pi$ is a semi-conjugacy, i.e. $\pi \sigma_{t}^{r}=\phi_{t} \pi$.

(iii) The map $\pi$ preserves the maximal measures.

(iv) $\sigma^{r}$ and $\phi$ have the same topological entropy $h=h(\phi)=h\left(\sigma^{r}\right)$.

We shall denote the Hölder exponent of $\pi$ by $\alpha>0$, i.e. $\exists C>0$ such that $d_{M}(\pi(\underline{x}), \pi(\underline{x})) \leqq C(d(\underline{x}, \underline{y}))^{\alpha}, \forall \underline{x}, \underline{y} \in \Sigma_{A}$, where $d_{M}$ denotes the metric on $M$.

Let $S_{i}=\left\{\phi_{t} \pi(\underline{x}) \mid x_{0}=i, 0 \leqq t \leqq r(\underline{x}), i=1, \ldots, k\right\}$ Clearly $\Lambda=\bigcup_{i=1} S_{i}$ since $\pi$ is surjective.

Associated to each $S_{i}$ is a partition $\mathscr{F}_{i}^{\text {ss }}$. We take each element of $\mathscr{F}_{i}^{\text {ss }}$ to be a set $W_{\varepsilon}^{\text {ss }}(x) \cap S_{i}$, where $x \in S_{i}$ and $\varepsilon>0$ is small with respect to the manifold, but large with respect to the size of the sections $T_{i}$. Each element of $\mathscr{F}_{i}^{\text {ss }}$ can be written in the form $\phi_{t} \pi\left\{\underline{y} \in \Sigma_{A} \mid x_{n}=y_{n}\right\}, 0 \leqq t \leqq r(\underline{x})$, where $\underline{x} \in \Sigma_{A}$ with $x_{0}=i$.

In the special case that $M$ is a three dimensional manifold the boundaries $\hat{c} T_{i}$ (and hence the boundaries $\partial S_{i}$ ) take a special form. Each of the discs $D_{i}$ is two dimensional and $T_{i}$ is a "rectangle" whose one dimensional sides lie in either strong stable or weak unstable leaves. We can assume that there are only finitely many orbits (which must therefore all be closed) which are confined to $\bigcup_{i} \partial S_{i}$ for all time. This only requires a slight modification to the construction in [4] (and in particular the lemma on p. 456). We need only require that the projection of strong stable manifolds onto each rectangle $T_{i}$ should be carried forward into the interior of rectangles sufficiently far in the future, and similarly for unstable manifolds projected onto $T_{i}$ on flowing backwards in time. Bowen first constructs a Markovian cover and then refines it to a Markov partition. The additional property described above is preserved under this procedure. Thus the forward image of the unstable part of the boundaries $\partial T_{i}$ eventually meet $\bigcup_{i} \partial T_{i}$ transversely, i.e. in a finite set of points. Similarly for the backward images of the stable parts of the boundaries.

Fig. 1

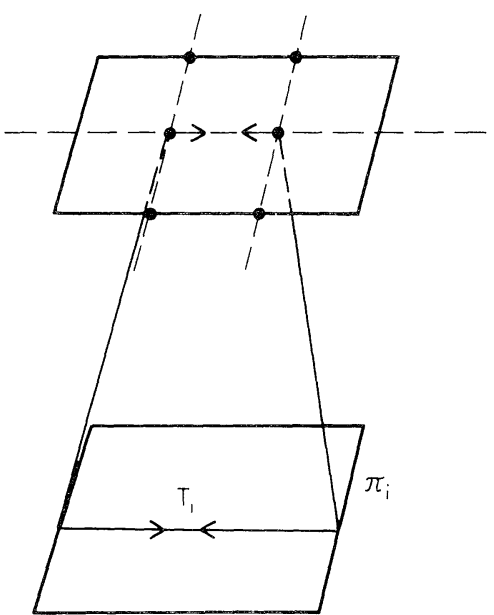


Remark. A slightly different system to Axiom $A$ flows are expanding semi-flows: Let $\phi_{t}: M \rightarrow M(t \geqq 0)$ be a semi-flow on a compact Riemannian manifold and let $T M=E^{0} \oplus E^{u}$, where $E^{0}$ is tangent to the flow direction and for $v \in E^{u}$ we have $\left\|D \phi_{t}(v)\right\| \geqq C e^{\lambda t}\|v\|$, for some $C, \lambda>0$. In a similar way to Axiom $A$ flows we can construct a symbolic dynamics (involving a one-sided subshift) and adapt the analysis of this paper.

\section{The Margulis Measure}

In 1970 Margulis published a paper in which he constructed a transverse measure for the strong-stable foliation for an Anosov flow [13]. In this section we shall present another approach to its construction which will generalise to a construction of "transverse distributions" in the next section.

Let $\{K\}$ be the family of compact transversals to the strong-stable foliation $\mathscr{F}^{\text {ss }}$. Let $v=\left\{v_{K}\right\}_{K}$ be a family of Borel measures with $v_{K}$ having support on $K$. Let $p: K \rightarrow K^{\prime}$ be a Borel isomorphism with $x, p(x) \in F_{x} \in \mathscr{F}^{\text {ss }}$. We call $v$ a transverse measure if $v_{K^{\prime}}(p A)=v_{K}(A)$ for all Borel sets $A \subseteq K$.

Margulis' approach was to construct $v$ as a linear functional on continuous functions $f$ of compact support $K$ transverse to the strong-stable foliation. He defined an equivalence relation on all such functions by $f \sim g$ if $f \circ p=g$, where $p: K^{\prime} \rightarrow K$ is a homeomorphism between the supports of $g$ and $f$ respectively such that $p(x) \in F_{x}$, where $x \in F_{x} \in \mathscr{F}^{\text {ss }}$. Margulis constructed a linear functional $v$ such that $v(f)=v(g)$ whenever $f \sim g$.

We shall now reconstruct this linear functional using symbolic dynamics. Much of this approach is implicit in [6].

To avoid confusion we shall restrict ourselves to the case of transitive three dimensional Anosov flows throughout the rest of this section. However, most of the analysis works in greater generality.

We construct a new space $\Sigma_{A}^{+}$defined by

$$
\Sigma_{A}^{+}=\left\{x \in \prod_{0}^{\infty}\{1, \ldots, k\} \mid A\left(x_{n}, x_{n+1}\right)=1 \text { for } n \geqq 0\right\}
$$

and a shift $\sigma: \Sigma_{A}^{+} \rightarrow \Sigma_{A}^{+}$by $(\sigma \underline{x})_{n}=x_{n+1}, n \geqq 0$ (we have retained the same notation as for $\sigma: \Sigma_{A} \rightarrow \Sigma_{A}$ defined in the preceding section).

We define a metric on $\Sigma_{A}^{+}$by $d(\underline{x}, \underline{y})=\sum_{n=0}^{+\infty} \frac{e\left(x_{n}, y_{n}\right)}{2^{n}}$, where

$$
e(i, j)=\left\{\begin{array}{lll}
1 & \text { if } & i \neq j \\
0 & \text { if } & i=j
\end{array} .\right.
$$

With this metric $\Sigma_{A}^{+}$is a compact, zero-dimensional space and $\sigma: \Sigma_{A}^{+} \rightarrow \Sigma_{A}^{+}$is a local homeomorphism.

Given $\alpha>0$ let $\mathscr{H}^{\alpha}=\mathscr{H}^{\alpha}\left(\Sigma_{A}^{+}, \mathbb{C}\right)$ denote those functions $f: \Sigma_{A}^{+} \rightarrow \mathbb{C}$ which are Hölder continuous with Hölder exponent $\alpha$. This function space becomes a Banach algebra with respect to the norm.

$$
\|f\|=\|f\|_{\infty}+\sup \left\{\frac{|f(\underline{x})-f(\underline{y})|}{[d(\underline{x}, \underline{y})]^{x}} \mid \underline{x} \neq \underline{y}\right\} .
$$


(The first term on the right-hand side is the supremum norm, and the second is the best Hölder constant for the exponent $\alpha$.)

Recall that $r: \Sigma_{A} \rightarrow \mathbb{R}$ was a function determined by its future co-ordinates [i.e. $r(x)=r(y)$ if $x_{n}=y_{n}$ for $\left.n \geqq 0\right]$ and therefore it can be considered as an element of $C\left(\Sigma_{A}^{+}, \mathbb{R}\right)$. In fact, $r \in \mathscr{H}^{\alpha}$ also, for an appropriate choice of $\alpha>0$ [4].

Let $h>0$ be the topological entropy of the flow $\phi_{t}: M \rightarrow M$. We assume the matrix $A$ to be aperiodic.

We define the Ruelle operator $L_{h r}: \mathscr{H}^{\alpha} \rightarrow \mathscr{H}^{x}$ by

$$
\left(L_{h r} g\right)(\underline{x})=\sum_{\underline{y}: \sigma v=\underline{x}} g(y) \exp -h r(y) .
$$

This is a bounded linear operator with a dual operator $L_{h r}^{*}:\left(\mathscr{H}^{\alpha}\right)^{*} \rightarrow\left(\mathscr{H}^{\alpha}\right)^{*}$ given by $\left(L_{h r}^{*} l\right)(g)=l\left(L_{h r} g\right)$. We summarise some of its relevant properties in the following proposition.

Proposition 2 (Ruelle Operator Theorem) (cf. [23, Sect. 5]).

(i) $L_{h r}$ has an eigenvalue at 1 with a strictly positive eigenvector in $\mathscr{H}^{\alpha}$.

(ii) The rest of spectrum of $L_{h r}$ is contained in a disc of strictly smaller radius than 1.

(iii) There exists a (positive) measure $\mu$ such that $L_{h r}^{*} \mu=\mu$.

There are some slight differences between the statement above and the usual version. However, the form that Proposition 2 takes can be easily derived from [23]. [In particular, to show part (i) we need only invoke Abramov's theorem.]

Corollary 2.1. $d \mu \sigma / d \mu=\exp h r$.

Proof. Given $\underline{x} \in \Sigma_{A}^{+}$we let $C_{n}=\left\{\underline{y} \mid x_{i}=y_{i}, 0 \leqq i \leqq n-1\right\}$ and let $\chi_{C_{n}}$ be the characteristic function for $C_{n}$. Then,

$$
\begin{aligned}
\mu\left(C_{n}\right) & =\int \chi_{C_{n}}(\underline{z}) d \mu(\underline{z}) \\
& =\int\left(L_{h r} \chi_{C_{n}}\right)(\underline{z}) d \mu(\underline{z}) \\
& =\int \sum_{i: A\left(i, z_{0}\right)=1} \chi_{C_{n}}(\underline{i z}) \cdot \exp -h r(\underline{i z}) d \mu(\underline{z}),
\end{aligned}
$$

where $\underline{i z}$ denotes the sequence $\left(i, z_{0}, z_{1}, z_{2}, \ldots\right)$. By the continuity of $r$ we have that for all $\varepsilon>0$ we can find $N>0$ so that

$$
\mu\left(\sigma C_{n}\right) e^{-(h r(\underline{x})+\varepsilon)} \leqq \mu\left(C_{n}\right) \leqq \mu\left(\sigma C_{n}\right) e^{-(h r(\underline{x})-\varepsilon)}
$$

for $n \geqq N$. (Here $\sigma C_{n}=\left\{\underline{z} \mid x_{i+1}=z_{i}, 0 \leqq i \leqq n-1\right\}$.) Thus $(d \mu \sigma / d \mu)(\underline{x})=\operatorname{exphr}(\underline{x})$ by letting $\varepsilon \rightarrow 0$.

Recall that we can cover $M$ with compact sets $S_{i}$ each equipped with a partition $\mathscr{F}_{i}^{\text {ss }}$ constructed using the strong stable foliation.

Let $S_{i}^{+}=\left\{(\underline{x}, t) \in \Sigma_{A}^{+} \times \mathbb{R} \mid x_{0}=i, 0 \leqq t \leqq r(\underline{x})\right\}$. We define a map $\pi_{i}^{+}: S_{i}^{+} \rightarrow S_{i} / \sim$ by $\pi_{i}^{+}(\underline{x}, t)=[\pi(\hat{x}, t)]$, where $x \sim y$ if $x$ and $y$ are in the same element of $F_{i}^{\mathrm{ss}},[]$ is an equivalence class in $S_{i}$, and $\underline{\hat{x}} \in \Sigma_{A}$ is any element with $\hat{x}_{n}=x_{n}$ for $n \geqq 0$.

Let $S^{+}=\bigcup_{i=1}^{k} S_{i}^{+}$and define $\pi^{+}: S^{+} \rightarrow \prod_{i=1}^{k} S_{i} / \sim$ by $\left.\pi^{+}\right|_{S_{i}^{+}}=\pi_{i}^{+}$. 
Let $K$ be a compact transverse section to $F^{\text {ss }}$ which supports a continuous (realvalued) function $f$. We shall assume initially that $K$ lies in some leaf of the strongstable foliation, i.e. $K \cong W^{\mathrm{wu}}(x)$ for some $x \in M$.

Let $K_{i}=K \cap S_{i}, i=1, \ldots, k$. We shall assume that the weak unstable manifold $W^{\mathrm{wu}}(x)$ containing $K$ satisfies $W_{\varepsilon}^{\mathrm{wu}}(z) \cap S_{i}=\overline{W_{\varepsilon}^{\mathrm{wu}}(z) \cap \operatorname{int} S_{i}}$ for $z \in W^{\mathrm{wu}}(x) \cap S_{i}$, $i=1, \ldots, k$. This is certainly true for a dense family of weak unstable leaves $W^{\mathrm{wu}}(x)$. There is a natural map $q_{i}: K_{i} \rightarrow S_{i} / \sim$. Without loss of generality we can assume that $K$ is sufficiently small (on the scale of the manifold $M$ ) that each $q_{i}$ is injective.

We define $\hat{f}: S^{+} \rightarrow \mathbb{R}$ by $\hat{f}(\underline{x}, t)=f \circ q_{i}^{-1} \circ \pi^{+}(\underline{x}, t)$ if $q_{i}^{-1} \pi^{+}(\underline{x}, t) \in K_{i}$ and $\hat{f}(x, t)=0$ otherwise. Choose $0<T<\inf r$. Define a linear functional on pairs $(f, K)$ by

$$
v(f)=\int_{\Sigma_{A}^{+}}^{r(\underline{x})} \int_{0}^{f} \hat{f}(\underline{x}, t) e^{-h t} d t d \mu(\underline{x}) .
$$

Observe that

$$
\int_{0}^{r(\underline{x})} \hat{f}(\underline{x}, t) e^{-h t} d t=\int_{0}^{r(\underline{x})-T} \hat{f}(\underline{x}, t) e^{-h t} d t+\sum_{i=1}^{k} \int_{r(\underline{x})-T}^{r(\underline{x})} \chi_{i}(\underline{x}) \hat{f}(\underline{x}, t) e^{-h t} d t
$$

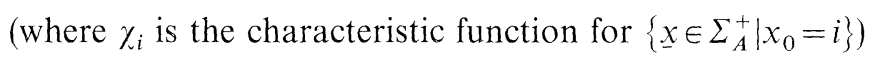

$$
\begin{aligned}
= & e^{h T} \int_{T}^{r(\underline{x})} \hat{f}(\underline{x}, t-T) e^{-h t} d t \\
& +e^{h T} e^{-h r(\varrho, \underline{x})} \sum_{i=1}^{k} \sum_{A(j, i)=1} \int_{0}^{T} \chi_{i}(\underline{x}) \hat{f}\left(\varrho_{j} \underline{x}, r\left(\varrho_{j} \underline{x}\right)-T+t\right) e^{-h t} d t,
\end{aligned}
$$

where $\varrho_{j}: \Sigma_{A}^{+} \rightarrow\left\{x \in \Sigma_{A}^{+} \mid x_{0}=j\right\}$ is the local inverse to the shift $\sigma$.

For brevity we shall write $\widehat{f^{0}}(\underline{x})=\int_{T}^{r(\underline{x})} \hat{f}(\underline{x}, t-T) e^{-h t} d t$ and

$$
\widehat{f^{i}}(\underline{x})=\int_{0}^{T} \chi_{i}(\sigma \underline{x}) \hat{f}(\underline{x}, r(\underline{x})-T+t) e^{-h t} d t .
$$

Then (3.2) becomes $e^{-h t}\left(\widehat{f^{0}}(\underline{x})+\sum_{i=1}^{k} L_{h r} \widehat{f^{i}}(\underline{x})\right)$. Therefore we can rewrite (3.1) as

$$
e^{h T}\left(\widehat{f^{0}}(\underline{x})+\sum_{i=1}^{k} L_{h r} \widehat{f^{i}}(\underline{x})\right) .
$$

Finally we have that

$$
v(f)=e^{h T} \int_{\Sigma_{A}^{+}}\left(\widehat{f^{0}}(\underline{x})+\sum_{i=1}^{k} L_{h r} \widehat{f^{i}}(\underline{x})\right) d \mu(\underline{x})=e^{h T} \int_{\Sigma_{A}^{+}}\left(\widehat{f^{0}}(\underline{x})+\sum_{i=1}^{k} \widehat{f^{i}}(\underline{x})\right) d \mu(\underline{x}),
$$

since by Proposition 2 (iii) we have that $\int_{\Sigma_{A}^{+}} L_{h r} \widehat{f^{i}} d \mu=\int_{\Sigma_{A}^{+}} \widehat{f^{i}} d \mu$. By the additional assumption we put on $W^{\text {wu }}\left(\underline{x}\right.$ ) (the weak-unstable leaf containing both $K$ and $\phi_{T} K$ ) we have that

$$
\int_{0}^{r(x)} \widehat{f \phi}-T(\underline{x}, t) e^{-h t} d t=\widehat{f}(\underline{x})+\sum_{i=1}^{k} \widehat{f^{i}}(\underline{x})
$$


and so by (3.3) we have that $v(f)=e^{h T} v\left(f \phi_{-T}\right)$. [By replacing $f$ by $f \phi_{T}$ gives $v\left(f \phi_{I}\right)$ $=e^{h T} v(f)$.]

To extend this to arbitrary $t>0$ choose $T=t / n<\inf r$, for a sufficiently large positive integer $n$. Then $v\left(f \phi_{t}\right)=v\left(f \phi_{n T}\right)=e^{h T} v\left(f \phi_{(n-1) T}\right)$. The result follows by induction.

We want to show that for $f \sim g$ then $v(f)=v(g)$. If $p: K^{\prime} \rightarrow K$ is a homeomorphism with $x, p(x) \in S_{i} \cap W_{\varepsilon}^{\text {ss }}(x)$ [for some $i=i(x)$ ] then this is clearly true by the construction. More generally we proceed as follows. Since we have assumed that $M$ is three dimensional there are at most a finite number of (closed) orbits intersecting $\bigcup_{i=1}^{k} \partial T_{i}$. Furthermore since $\pi$ is bounded to one these correspond to a finite number $N$, say, of sequences $\left\{\underline{x}^{(i)}\right\}_{i=1}^{N}$ in $\Sigma_{A}^{+}$. For each $i=1, \ldots, N$ let

$$
C^{i}=C^{i}(Q)=\left\{\underline{z} \in \Sigma_{A}^{+} \mid z_{j}=x_{j}^{(i)} \text { for } j=1, \ldots, Q\right\} \text { and } C=\bigcup_{i=1}^{k} C^{i},
$$

where $Q$ is a large integer. The set

$$
K(Q)=\bigcup_{i=1}^{k} q_{i}^{-1} \circ \pi_{i}^{+}\{(\underline{z}, t) \mid \underline{z} \in C, 0 \leqq t \leqq r(\underline{x})\}
$$

is a neighbourhood in $K$ around the projection onto $K$ of the closed orbits intersecting $\bigcup_{i=1}^{k} \partial T_{i}$. Corresponding to $f_{K(Q)}$ and $f_{K-K(Q)}$, we have

$$
v(f)=\int_{C} \int_{0}^{r(\underline{x})} \hat{f}(\underline{x}, t) e^{-h t} d t d \mu(\underline{x})+\int_{\Sigma_{A}^{+}-C}^{r(\underline{x})} \hat{f}(\underline{x}, t) e^{-h t} d t d \mu(\underline{x}),
$$

and

$$
v(g)=\int_{p(C)} \int_{0}^{r(\underline{x})} g(\underline{x}, t) e^{-h t} d t d \mu(\underline{x})+\int_{\Sigma_{A}^{+}} \int_{-p(C)}^{r(\underline{x})} \int_{0}^{r} g(\underline{x}, t) e^{-h t} d \mu(\underline{x}) .
$$

We can equate the second terms in (3.4) and (3.5). To see this let $x=\pi^{+}(x, t)$ and $y=\pi^{+}(y, s)$, where $x \notin C$ then there exists $t_{0}>0$ such that $\phi_{t_{0}}(x), \phi_{t_{0}}(y)$ both lie in the same set $S_{i}\left(i=i\left(x, t_{0}\right)\right)$. This is because the orbit of $x$ cannot always be confined to $k$

$\bigcup_{i=1} \partial S_{i}$ else it would correspond to a periodic orbit, contradicting our assumption that $x \notin C$. If $x$ and $y$ are sufficiently close, then when $\phi_{t_{0}} x \in \operatorname{int} S_{i}$, say, then we can assume that $\phi_{t_{0}} y$ lies in the same set. Furthermore, we can choose a neighbourhood $C(x) \times(t-\varepsilon, t+\varepsilon)$ about $(x, t)$ such that this remains true for all points in this set. By considering $\phi_{t^{\prime}} C(\underline{x}) \times(t-\varepsilon, t+\varepsilon)$ for a range of values of $t^{\prime}$ close to $t_{0}$ we can establish the equality of the second terms in (3.4) and (3.5) with $C$ replaced by $C(\underline{x})$. But by a compactness argument we can cover $C$ by only finitely many such $C(x)$. Summing gives the desired result.

In remains to deal with the first terms in (3.4) and (3.5). We shall show that these terms tend to zero as $Q \rightarrow+\infty$. Since $\mu$ is a positive measure we have

$$
\left|\int_{C} \int_{0}^{r(\underline{x})} \hat{f}(\underline{x}, t) e^{-h t} d t d \mu(\underline{x})\right| \leqq \mu(C)\|\hat{f}\|_{\infty}\|r\|_{\infty} .
$$


However, $\mu(C) \leqq \mu\left(\sigma^{Q} C\right) \cdot(\|d \mu / d \mu \sigma\|)_{\infty}^{Q}$ which tends to zero as $Q$ tends to infinity since by Corollary 2.1 we know that $\|d \mu / d \mu \sigma\|_{\infty} \leqq \exp -(\inf r)<1$.

The condition we imposed on $W^{\text {wu }}(x)$ is unimportant, since we have established the result on a dense set of transversals. Given a function supported on any other compact transversal we can project it onto a transversal of the form considered above (after localising with a partition of unity if necessary).

We have now established a version of the following theorem due to Margulis [13]:

Theorem 1 (Margulis). Let $\phi_{t}: M \rightarrow M$ be a (three dimensional) transitive Anosov flow with topological entropy $h>0$. There exists a transverse measure to the strongstable foliation $\mathscr{F}^{\mathrm{ss}}$ of $M$ which satisfies $\phi_{t}^{*} v=e^{h t} v$.

Remark. If $F^{1}$ denotes the smooth 1 -forms on $M$, then a current is a continuous linear map $F^{1} \rightarrow \mathbb{C}$. Given a transverse measure $v$ there is an associated current, called the Ruelle-Sullivan current $C$ [25], defined as follows: Choose a cover for $M$ by interiors of the images of sets of the form $D_{i}^{T} \times D_{i}$, where $D_{i}^{T}$ corresponds to a transverse section and $D_{i}$ corresponds to neighbourhoods on each leaf. Assume that $w_{i}$ is a smooth 1 -form with support on $D_{i}^{T} \times D_{i}$, then we define

$$
C\left(w_{i}\right)=\int_{D_{l}^{T}}\left\{\int_{\{x\} \times D_{\imath}} w\right\} d v_{D_{l}^{T}}(x),
$$

and extend to all smooth 1 -forms using a partition of unity. (The inner integral is along leaves of the foliation and they are weighted in the outer integral by the transverse measure supported on $D_{i}^{T}$.) The integral on a leaf has to be with respect to an orientation. Ruelle and Sullivan showed this defines a closed current i.e. $C \circ d=0$ ( $d$ is the exterior derivative on forms) and so we can consider $[C] \in H^{2}(M, \mathbb{C}) \quad[25]$. The condition $\phi_{t}^{*} v=e^{h t} v$ gives that $\phi_{t}^{*}[C]=e^{-h t}[C]$, provided the orientation on leaves is respected by $\phi_{t}$. However, since $\phi_{t}$ is homotopic to the identity we have that, for $t>0, e^{-h t}$ cannot be an eigenvalue of $\phi_{t}^{*}$ and we conclude that $[C]=0$.

Following Schwartzman [26] (as interpreted by Sullivan [28]) we have the following: Given $x \in D$ let $\alpha$ be a loop formed by a segment of the foliation leaf starting at $x$ of length $L$ and with the ends linked by a geodesic. This gives $\left[\alpha_{L}\right] \in H_{1}(M, \mathbb{R})$. For $a \cdot a \cdot(v) x \in D$ we have $\left[\alpha_{L}\right] \rightarrow 0$ (corresponding to $[C]=0$ ).

\section{Rates of Mixing and Margulis Distributions}

In this section we shall recall some results on the rate of mixing of an Axiom $A$ flow and describe its connection with certain generalisations of the Margulis measure.

The basic problem is as follows: Let $\phi_{t}: \Lambda \rightarrow \Lambda$ be a weak-mixing Axiom $A$ flow and let $F, G: \Lambda \rightarrow \mathbb{C}$ be Hölder continuous and let $m$ be the maximal measure. It is known that $\int F \phi_{t} \cdot G d m \rightarrow \int F d m \cdot \int G d m$ as $t \rightarrow+\infty$. We want to describe the rate of this convergence

We set

$$
\varrho(t)= \begin{cases}\int F_{i} \cdot G d m-\int F d m \cdot \int G d m & \text { for } t \geqq 0 \\ 0 & \text { for } t \leqq 0\end{cases}
$$



and approach the problem by studying its Fourier transform $\varrho(s)=\int_{-\infty}^{+\infty} e^{i s t} \varrho(t) d t$.
One result in this direction is the following:

Proposition $3[16,21]$. The function $\varrho(s)$ has a meromorphic extension to a half-plane $\operatorname{Im}(s)>-c($ for some $c>0)$.

The behaviour of $\varrho(t)$ is considerably influenced by the domain of $\varrho(s)$ and, in particular, by the location of its poles. These in turn are closely related to the Ruclle operator through the symbolic dynamics.

For $z \in \mathbb{C}$ we have the Ruelle operator $L_{z r}: \mathscr{H}^{\alpha} \rightarrow \mathscr{H}^{\alpha}$, where $\mathscr{H}^{\alpha}$ denotes the Banach algebra of $\alpha$-Hölder continuous complex valued functions $f: \Sigma_{A}^{+} \rightarrow \mathbb{C}$.

The following proposition was proved in [17].

Proposition 4 (Complex Ruelle Operator Theorem). There exists $C>0$ such that for $\operatorname{Re}(z)>h-C$ the essential spectrum of $L_{z r}: \mathscr{H}^{\alpha} \rightarrow \mathscr{H}^{\alpha}$ is contained in the disc $\{w \in \mathbb{C}|| w \mid<1\}$.

We recall that the essential spectrum of an operator is that part of the spectrum that remains after removing isolated eigenvalues of finite multiplicity which correspond to finite dimensional eigenspaces.

The relationship between the poles for $\hat{\varrho}(s)$ and the spectrum of the operator $L_{z r}$ is given by the following (cf. $[16,21]$ ).

Proposition 5. If we write $z=h-i$ s then the following are equivalent in the range $\operatorname{Im}(s)>-c($ for some $c>0)$.

(a) For $z=z_{0}, 1$ is in the spectrum of $L_{z r}: \mathscr{H}^{\alpha} \rightarrow \mathscr{H}^{\alpha}$ (and $\lambda_{i}\left(z_{0}\right)=1$ is an isolated eigenvalue of multiplicity $m \geqq 1$ ).

(b) For $s=s_{0}$, $\varrho(s)$ has a pole.

As before we shall now confine our attention to three dimensional transitive Anosov flows.

An important feature of Anosov flows is that the strong stable foliation $\mathscr{F}^{\text {ss }}$ is Holder continuous. We shall assume that the Holder exponent is 1 .

Let $F$ be a complex valued function which is compactly supported on a weak unstable leaf $W^{\mathrm{wu}}(x)$. Furthermore we assume that $F$ is $\alpha$-Holder continuous. We shall call two functions $F, G$ related if there exists a homeomorphism $p: K^{\prime} \rightarrow K$ between the compact supports of $F$ and $G$ with $F \triangleright p=G$ such that if $x \in F_{x} \in \mathscr{F}^{\text {ss }}$ then $p(x) \in K^{\prime} \cap W_{\varepsilon}^{\text {ss }}(x)$. We shall call a linear functional $v$ on such functions a "transverse distribution" if $v(F)=v(G)$ whenever $F \sim G$. (We should observe that if we want all related functions to have the same Holder exponent it will generally be necessary to assume that the Holder exponent of the strong-stable foliation is unity.)

Given $F$ as above we define $\hat{F}:\left\{(\underline{x}, t) \in \Sigma_{A}^{+} \times \underset{\mathbb{R}}{0} \mid 0 \leqq t \leqq r(x)\right\} \rightarrow \mathbb{C}$ as in the previous section. We then define $f \in \mathscr{H}^{\alpha}\left(\Sigma_{A}^{+}, \mathbb{C}\right)$ by $f(\underline{x})=\int_{0}^{r(\underline{x})} \hat{F}(\underline{x}, t) e^{z t} d t$, where $z \in \mathbb{C}$.

Assume that 1 is an isolated eigenvalue for $L_{z r}: \mathscr{H}^{\alpha} \rightarrow \mathscr{H}^{\alpha}$ with $L_{z r}^{*} \mu=\mu$, with $\mu \in\left[\mathscr{H}^{\alpha}\left(\Sigma_{A}^{+}, \mathbb{C}\right)\right]^{*}$. 
Without loss of generality we shall assume, as in the previous section, that $W_{\varepsilon}^{\mathrm{wu}}(z) \cap S_{i}={\overline{W_{\varepsilon}^{\mathrm{wu}}}(z) \cap \operatorname{int} S_{i}}_{i}$ for $z \in K \cap S_{i}$. Under these conditions we define $v(F)$ $=\mu(f)$.

Let $0<T<\inf r$.

Let $g(\underline{x})=\int_{0}^{r(x)} \widehat{F \phi}_{T} e^{z t} d t$, then

$$
\begin{aligned}
g(\underline{x})= & \int_{0}^{r(x)} \hat{F} \phi_{T}(\underline{x}, t) e^{z t} d t=\int_{T}^{r(\underline{x})} \hat{F}(\underline{x}, t-T) e^{z t} d t \\
& +\sum_{i} \sum_{A(j, i)=1} \int_{0}^{T} \chi_{i}(\underline{x}) \hat{F}\left(\varrho_{j} \underline{x}, r\left(\varrho_{j} \underline{x}\right)-T+t\right) e^{z t} d t
\end{aligned}
$$

(where $\chi_{i}$ is the characteristic function for $\left\{\underline{x} \mid x_{0}=i\right\}$ and $\varrho_{j}:\left\{x \mid x_{0}=j\right\} \rightarrow \Sigma_{A}^{+}$is the local inverse to $\sigma: \Sigma_{A}^{+} \rightarrow \Sigma_{A}^{+}$). Therefore:

$$
\begin{aligned}
g(\underline{x})= & e^{z T} \int_{0}^{r(\underline{x})-T} \hat{F}(\underline{x}, t) e^{z t} d t \\
& +\sum_{i} \sum_{A(j, i)=1}\left\{\int_{T-r(\underline{\underline{o}}, \underline{x})}^{r(\underline{x})} \chi_{i}(\underline{\underline{x}}) \hat{F}\left(\varrho_{j} \underline{\underline{x}}, t\right) e^{z t} d t\right\} e^{z T} e^{-z r\left(\varrho_{j} \underline{x}\right)} \\
= & e^{z T}\left[f^{0}(\underline{x})+\sum_{i} \sum_{A(j, \underline{i})=1} f^{i}\left(\varrho_{j} \underline{x}\right) e^{-z r\left(\varrho_{j} \underline{x}\right)}\right]
\end{aligned}
$$

$\left[\right.$ where $\left.f^{0}(\underline{x})=\int_{0}^{r(\underline{x})-T} \hat{F}(\underline{x}, t) e^{z t} d t ; f^{i}(\underline{y})=\int_{T-r(\underline{y})}^{r(\underline{y})} \chi_{i}(\sigma \underline{y}) \hat{F}(\underline{y}, t) e^{z t} d t\right]$. In particular, $g(\underline{x})$ $=e^{z T}\left(f^{0}(\underline{x})+\sum_{i} L_{s r} f^{i}(\underline{x})\right)$ and

$$
\mu(g)=e^{z T}\left(\mu\left(f^{0}\right)+\sum_{i} \mu\left(f^{i}\right)\right)=e^{z T} \cdot \mu\left(f^{0}+\sum_{i} f^{i}\right)=e^{z T} \cdot \mu(f) .
$$

Thus we may conclude that $v\left(F \phi_{T}\right)=e^{z T} \cdot v(F)$.

The argument to show that related functions $F$ and $G$ give the same value $v(F)$ $=v(G)$ follows the same lines as the proof for the Margulis measure in the previous section. The only additional feature is in proving that the contribution to $\mu(f)$ and $\mu(g)$ "from $C$ " are the same. Assume that $f\left(\underline{x}^{i}\right)=0$. Since $f$ is Holder continuous we have $\left\|\left.f\right|_{C^{i}}\right\|_{\infty} \rightarrow 0$ as $Q \rightarrow+\infty$. Furthermore we have that $\mu$ is a bounded linear functional on $\mathscr{H}^{\alpha^{\prime}}\left(\Sigma_{A}^{+}, \mathbb{C}\right)$ for some $\alpha^{\prime}<\alpha$. (Changing the exponent $\alpha$ has the effect of changing the constant $c$ in Proposition 3.) If \|\|$_{\alpha}$ and \|\|$_{\alpha^{\prime}}$ are the corresponding Holder semi-norms on for the spaces of Holder continuous functions with exponents $\alpha$ and $\alpha^{\prime}$, respectively, then $\left\|\left.f\right|_{C^{\prime}}\right\|_{\alpha^{\prime}} \leqq\|f\|_{\alpha}\left(\frac{1}{2}\right)^{\left(\alpha-\alpha^{\prime}\right) Q}$. Thus $\left|\mu\left(\left.f\right|_{C^{2}}\right)\right| \leqq\|\mu\|_{\alpha^{\prime}}\left(\left\|\left.f\right|_{C^{2}}\right\|_{\alpha^{\prime}}+\left\|\left.f\right|_{C^{*}}\right\|_{\infty}\right) \rightarrow 0$ as $Q \rightarrow+\infty$. Hence $v(F)=v(G)$.

If $f\left(\underline{x}^{(i)}\right) \neq 0$ then we replace $f$ by $f-f\left(\underline{x}^{(i)}\right)$. The limit then becomes $f\left(x^{(i)}\right) \mu(1)$, and so again the expressions $v(F)$ and $v(G)$ can be seen to be the same by taking limits.

As before we can remove the condition on $W^{\mathrm{wu}}(x)$ by projecting onto one of the dense family of leaves satisfying this condition. (Again we note that unless we assume that the Holder coefficient for the strong-stable foliation is unity then this may involve a modification in the exponent.)

We summarize below 
Theorem 2. Let $\phi_{t}: M \rightarrow M$ be a three dimensional Anosov flow whose strong stable foliation has Holder exponent unity. If $s=s_{0}$ is a pole for the complex function $\varrho(s)$ ( for $\operatorname{Im}(s)>-C$, say) then there exists a linear functional $v$ on the class of $\alpha$-Holder continuous transverse functions, for $\alpha \leqq 1$, such that $\phi_{t}^{*} v=e^{z t} v$, where $z=h$-is (i.e. a transverse distribution).

By analogy with the case of Margulis measures we call the transverse distributions in the above theorem Margulis Distributions.

The assumption that the foliations should have Holder exponent unity will not prove necessary in applications, since we shall generally need to deal with a finite family of transversals. We should remark that it is true for geodesic flows for surfaces of strictly negative curvature where the foliation is even $C^{1}$.

\section{Location of Poles}

We have analysed the meromorphic domains of Fourier transforms of correlation functions (for the maximal measure) and characterised the poles in terms of transverse Margulis distributions. We now want to use this to get concrete information on the location of poles during this section and the next. Let $\phi_{t}: M \rightarrow M$ be a transitive three dimensional Anosov Flow.

Let $v$ be a $C^{\alpha}$ Margulis distribution transverse to the strong-stable foliation $\mathscr{F}^{\text {ss }}$. The Banach space of complex $C^{\alpha}$-forms tangent to the foliation will be denoted by $C_{s}^{\alpha}$, and let $\left(C_{s}^{\alpha}\right)^{*}$ denote its dual. We want to define an element $m$ in $\left(C_{s}^{\alpha}\right)^{*}$ using the Margulis distribution $v$. Given a $C^{\alpha} 1$-form $w$ tangent to the foliation, assume that $w$ has support (as in Sect. 3) on $D^{T} \times D$, where $D^{T}$ supports the transverse distribution $v_{D}$. We have a $C^{\alpha}$-function defined on $D^{T}$ by $x \rightarrow \int_{D \times\{x\}} w$, i.e. integrating the form across the leaf $D \times\{x\}$. We define $m \in\left(C_{s}^{\alpha}\right)^{*}$ by

$$
m(w)=\int_{D^{T}}\left\{\int_{D \times\{x\}} w\right\} d v_{D^{T}}(x)
$$

and a $C^{\infty}$-partition of unity. (This definition is obviously motivated by the RuelleSullivan current.) The bundles $E^{u}$ and $E^{s}$ are automatically oriented and so $x \rightarrow \int_{D \times\{x\}} w$ can be defined with a consistent choice of sign.

By definition we know that $\phi_{t}^{*} v=e^{z t} v$. We therefore have that $\phi_{t}^{*} m=e^{-z t} m$, i.e. $m$ is an eigenfunction for $\phi_{t}^{*}:\left(C_{s}^{\alpha}\right)^{*} \rightarrow\left(C_{s}^{\alpha}\right)^{*}$ with eigenvalue $e^{-z t}$. (We shall assume that $t>0$ is fixed throughout this argument.)

We know that $\phi_{t}^{*}:\left(C_{\mathrm{s}}^{\alpha}\right)^{*} \rightarrow\left(C_{s}^{\alpha}\right)^{*}$ is the dual operator to the $\phi_{t}$-induced operator $\phi_{t^{*}}: C_{s}^{\alpha} \rightarrow C_{s}^{\alpha}$, and as such shares the same spectrum. We then conclude that $e^{-z t}$ lies in the spectrum of $\phi_{t^{*}}: C_{s}^{\alpha} \rightarrow C_{s^{*}}^{\alpha}$ We state this as a theorem:

Theorem 3. Let $\phi_{t}: M \rightarrow M$ be a transitive three dimensional Anosov flow and let $\phi_{t^{*}}: C_{\mathrm{s}}^{\alpha} \rightarrow C_{\mathrm{s}}^{\alpha}$ be the induced operator on $C^{\alpha}$-forms tangent to the strong stable foliation.

There exist $C, \alpha>0$ such that if $s=s_{0}, \operatorname{Im}(s)>-c$, is a pole for $\hat{\varrho}(s)$ (with respect to the maximal measure), then $e^{-z_{0} t}$ is in the spectrum of $\phi_{t^{*}}$, where $z_{0}=h-i s_{0}$. (There is a similar version of this result for $\phi_{-t^{*}}: C_{u}^{\alpha} \rightarrow C_{u}^{\alpha}$ for the unstable foliation.) 
Let $\phi_{t}: M \rightarrow M$ be an Anosov flow for which spectrum of $\phi_{t^{*}}: C_{s}^{\alpha} \rightarrow C_{s}^{\alpha}$ is contained in a single circle. We call such a flow homogeneous. Examples of homogeneous flows are geodesic flows on surfaces of constant negative curvature and suspensions by constant functions of hyperbolic toral automorphisms $f: T^{2}-T^{2}$. To see this we have only to apply the standard equation on the transformation of forms under the action of a diffeomorphism. This corresponds to scalar multiplication by expht and the result follows from the spectral radius theorem.

A flow is weak-mixing if there are no non-trivial solutions to the equation $F\left(\phi_{t}\right)$ $=e^{i a t} F$, with $a>0$ and $F \in C(M)$.

A direct application of Theorem 3 gives:

Corollary 3.1. For any homogeneous weak-mixing flow, $\varrho(s)$ is analytic for $\operatorname{Im}(s)>-C$ (for some $C>0$ ).

This result is known already for geodesic flows on surfaces of constant negative curvature (cf. [20]).

\section{Geodesic Flows}

In this section we restrict our attention to the very important case of geodesic flows on manifolds of negative (sectional) curvature.

Let $M$ be a compact manifold with Riemannian metric $\langle$,$\rangle of negative$ sectional curvatures. We define a geodesic flow on the unit tangent bundle in the usual way: Given $(x, v) \in R_{1} M$, let $\gamma: \mathbb{R} \rightarrow M$ be the unique unit speed geodesic through $x$ in the direction $v$ at time $t=0$ [i.e. $\gamma(0)=x, \dot{\gamma}(0)=v)$ then set $\phi_{t}(x, v)$ $=(\gamma(t), \dot{\gamma}(t))]$. This defines the geodesic flow $\phi_{t}: T_{1} M \rightarrow T_{1} M$.

To understand better $D \phi_{t}: T\left(T_{1} M\right) \rightarrow T\left(T_{1} M\right)$ we follow Eberlein [9]. The map $\pi: T M \rightarrow M, \pi(x, v)=x$ gives the derivative $D \pi: T_{(x, v)}(T M) \rightarrow T_{x} M$. We can construct a second linear map $K: T_{(x, v)}(T M) \rightarrow T_{x} M$ by first choosing for $\xi \in T_{(x, v)}(T M)$ a curve $Z:(-\varepsilon, \varepsilon) \rightarrow T M$ tangent to $\xi$ at time $t=0$. We then have a curve $\alpha=\pi \circ Z:(-\varepsilon, \varepsilon) \rightarrow M$ and set $K \xi=\nabla_{\alpha} Z$, i.e. the covariant derivative of $Z$ along $\alpha$ at time $t=0$. It is known that for all $(x, v) \in T M$ we can decompose $T_{(x, v)}(T M)$ as $T_{(x, v)}(T M)=(\operatorname{Ker} D \pi) \oplus(\operatorname{Ker} K)$. The Riemannian metric $\langle,\rangle_{x}$ for $M$ then prompts the definition of a metric for $T M$ by $\langle\xi, \eta\rangle_{(x, v)}=\langle D \pi \xi, D \pi \eta\rangle_{x}+\langle K \xi, K \eta\rangle_{x}$, for $\xi, \eta \in T_{(x, v)}(T M)$. We proceed to consider the evolution of the flow $\phi_{t}$. For every point $(x, v) \in T M$ we have an associated geodesic $\gamma$ with $\gamma(0)=x$ and $\dot{\gamma}(0)=v$. Given any $\xi \in T_{(x, v)}(T M)$ we associate with it the Jacobi field $Y_{\xi}$ on $\gamma$ such that $Y_{\xi}(x)=D \pi \xi$, $\nabla_{\alpha} Y(x)=K \xi$. This map $\xi \rightarrow Y_{\xi}$ gives a linear isomorphism from $T_{(x, v)}(T M)$ to the Jacobi fields on $\gamma[$ for $(x, v)]$. The map $D \phi_{t}: T(T M) \rightarrow T(T M)$ is related to this geometric construction by: $D \pi D \phi_{t}(\xi)=Y_{\xi}(\gamma(t))$ and $D K D \phi_{t}(\xi)=\left(\nabla_{\gamma} Y_{\xi}\right)(\gamma(t))$.

To understand this characterisation of $D \phi_{t}$, we consider the following view of Jacobi fields (due to Green [10]). For the geodesic $\gamma$ let $e_{1}(t), \ldots, e_{n}(t)$ be a system of parallel orthonormal vector fields along $\gamma$ with $\gamma$ tangent to $e_{n}(t)$ at $t=0$. (This is called an adapted frame field.) A vector field $Y$ on $\gamma$ can be identified with $(n-1)$ tuple $\left(y_{1}(t), \ldots, y_{n-1}(t)\right)$ of $C^{\infty}$ functions $y_{i}: \mathbb{R} \rightarrow \mathbb{R}$, where $Y(t)=\sum_{i} y_{i}(t) e_{i}(t)$. The covariant derivative becomes $\nabla_{\gamma} Y=\sum_{i} y_{i}^{\prime}(t) e_{i}(t)=Y^{\prime}(t)$. 
Let $R$ be the curvature tensor for $M$ (i.e. $R(X, Y) Z=\left[\nabla_{x} Y, \nabla_{Y} X\right] Z-\nabla_{[x, y]} Z$ ), then we can define an $(n-1) \times(n-1)$ matrix $R(t)=\left(R_{i j}(t)\right)$ by

$$
R_{i j}(t)=\left\langle R\left(e_{n}(t), e_{i}(t)\right) e_{n}(t), e_{j}(t)\right\rangle .
$$

A perpendicular Jacobi vector field on $\gamma$ is defined by $t \rightarrow Y(t) x$, where $x \in \mathbb{R}^{n-1}$ and $Y(t)$ is a solution of the matrix differential equation

$$
Y^{\prime \prime}(t)+R(t) Y(t)=0 \text {. }
$$

[For a surface this reduces to a single differential equation where $R(t)$ is the curvature at $\gamma(t)$.]

We can conclude from the above that smooth changes in the metric will cause a smooth change in the operator $D \phi_{t}: T\left(T_{1} M\right) \rightarrow T\left(T_{1} M\right)$ and $\phi_{t^{*}}: C^{\alpha} \rightarrow C^{\alpha}$ for the associated geodesic flows. (Here a Riemannian metric is a smooth section in the fibre bundle of positive definite inner products for the tangent space. A smooth change in the topology of sections should correspond to a smooth change in the curvature tensor and so to a smooth change in the Jacobi vector fields. Similarly a smooth change in the metric should give a smooth change in the derivative of the Jacobi vector fields. Together these induce a smooth change in $D \phi_{t}$ by the decomposition described above.) When the manifolds have constant sectional curvatures $K=-k^{2}$, then the flow is homogeneous and the spectrum is contained in a circle of radius exp $-t k$. For smooth perturbations in the metric the spectrum of $\phi_{t^{*}}: C_{s}^{\alpha} \rightarrow C_{s}^{\alpha}$ is contained in an annulus with inner and outer radii close to $\exp -h t$ [where $h=h(\phi)$ is the topological entropy of the flow].

We may assume that the width of the meromorphic extension is constant (since the factors that determine this - the roof function, the expansiveness of the flow, and the geometric constraints on the transverse sections - vary continuously with the metric cf. [17]). Together these two observations give the following.

Theorem 4. Let $\langle$,$\rangle be a metric of constant negative (sectional) curvature on a$ compact manifold and let $\langle,\rangle^{\prime}$ be a $C^{\infty}$-close metric, then the Fourier transform of a corresponding correlation function $\hat{\varrho}(s)$ has an analytic extension to a half-plane $\operatorname{Im}(s)>-\delta$, for some $\delta>0$.

\section{The Zeta Function}

So far we have confined ourselves to the relationship between Margulis distributions and the Fourier transform of the correlation function. This analysis is squarely based on the connection of the spectrum of the Ruelle operator with the meromorphic domain of the Fourier transform. However, there is a parallel theory for the meromorphic extension of a certain zeta function associated with the Axiom $A$ flow. Here the poles are again determined by the spectrum of the zeta function [17]. We shall now turn our attention to the relationship between this zeta function and Margulis distributions.

Let $\phi_{t}: M \rightarrow M$ be an Axiom $A$ flow and let $A$ be an attractor. For each closed orbit $\tau$ we denote its least period by $\lambda(\tau)$. We define the zeta function for $\phi$ by

$$
\zeta(s)=\prod_{\tau}(1-\exp -s \lambda(\tau))^{-1}
$$


where $s \in \mathbb{C}$. (The Euler product converges to a non-zero, analytic function for $R(s)>h[24]$.) We know the following about the domain of $\zeta(s)$ :

Proposition 6 [17]. The zeta function $\zeta(s)$ has a non-zero meromorphic extension to a half-plane $\operatorname{Re}(s)>h-c$, for some $c>0$. Furthermore, $s=s_{0}$ is a pole for $\zeta(s)$ if and only if 1 is an eigenvalue for $L_{-s_{0} r}: \mathscr{H}^{\alpha} \rightarrow \mathscr{H}^{\alpha}$, for some $\alpha>0$.

The constant $c>0$ is dictated not only by the constraints of the associated suspended flow but also by the need to take into account difficulties with closed orbits which pass through the boundaries of the transverse sections (although when $\operatorname{dim} M=3$ there are only finitely many such orbits). There is a simple pole at $s=h$ which corresponds to 1 being an eigenvalue for $L_{h r}$, by the Ruelle operator theorem. The condition that $\phi$ is weak-mixing is equivalent to $\zeta(s)$ being analytic on the line $\operatorname{Re}(s)=h$, except for $s=h$. If $\phi$ is not weak-mixing then $\zeta(s)$ has period $i$, for some $a>0$ [14].

Parallel to Theorem 3 for correlation functions we have the following:

Theorem 5. Let $\phi_{t}: M \rightarrow M$ be a transitive three dimensional Anosov flow and let $\phi_{t^{*}}: C_{s}^{\alpha} \rightarrow C_{s}^{\alpha}$ be the induced operator on $C^{\alpha}$-forms along the stable manifold. There exists $c>0$, such that if $s=s_{0}$, with $\operatorname{Re}(s)>h-c$, is a pole for $\zeta(s)$, then $e^{-s_{0} t}$ is in the spectrum of $\phi_{t^{*}}$.

The usefulness of this theorem depends on our information on the spectrum of $\phi_{t^{*}}$ as before. Corresponding to Corollary 3.1 we have:

Corollary 5.1. For any homogeneous flow $\zeta($ s) is analytic for $\operatorname{Re}(s)>h-c$ (for some $c>0)$, except for a simple pole at $s=h$ if $\phi$ is weak-mixing or simple poles at $h+$ nia $(n \in \mathbb{Z})$ if $\phi$ is not weak-mixing.

This is applicable to geodesic flows for surfaces of constant negative curvature and constant suspensions of hyperbolic toral automorphisms.

The most interesting case is that of the geodesic flow. In this context we have the following:

Theorem 6. Let $\langle$,$\rangle be a metric of constant negative curvature on a compact surface.$ For geodesic flows corresponding to metrics $\langle,\rangle^{\prime} C^{\infty}$ close to $\left\langle>\right.$ we have that $\zeta_{\langle,\rangle}(s)$ is analytic in a half plane $\operatorname{Re}(s)>h-c$, except for a simple pole at $s=h$, for some $c>0$ (where $h$ is the entropy of the geodesic flow for $\langle,\rangle^{\prime}$ ).

The case of constant curvature surfaces has been extensively studied cf., for example, [11].

Acknowledgements. I am pleased to acknowledge the debt this paper owes to my association with William Parry and David Ruelle, and the work of Rufus Bowen.

Some of the early work on this paper was done at IHES. I gratefully acknowledge their support, and the hospitality of N. H. Kuiper.

\section{References}

1. Anosov, D.V.: Geodesic flows on closed Riemannian manifolds with negative curvature. Proc. Steklov Inst. Math. 90, 1-235 (1967)

2. Anosov, D.V.: On tangent fields of transversal fibrations in $Y$-systems, Math. Zametki 2 , 539-548 (1967) 
3. Anosov, D.V., Sinai, Ya.G.: Some smooth ergodic systems. Russ. Math. Surv. 22 (5), 103-167 (1967)

4. Bowen, R.: Symbolic dynamics for hyperbolic flows, Am. J. Math. 95, 429-460 (1973)

5. Bowen, R.: Equilibrium states and the ergodic theory of Anosov diffeomorphisms. Lecture Notes in Mathematics, Vol. 470. Berlin, Heidelberg, New York: Springer 1975

6. Bowen, R., Marcus, B.: Unique ergodicity of horocycle foliations. Isr. J. Math. 26, 43-67 (1977)

7. Bowen, R., Ruelle, D.: The ergodic theory of Axiom A flows. Invent. Math. 29, 181-202 (1975)

8. Connes, A.: A survey of foliations and operator algebras. Proc. Symp. Pure Math. 38, $521-628$ (1982)

9. Eberlein, P.: When is a geodesic flow of Anosov type? I. J. Differ. Geom. 8, 437-463 (1972)

10. Green, L.W.: Remarks on uniformly expanding horocycle parameterisations. J. Differ. Geom. 13, 263-271 (1978)

11. Hejhal, D.A.: The Selberg trace formula and the Riemann zeta function. Duke Math: J. 43, 441-482 (1976)

12. Hirsch, M.W., Pugh, C.: Smoothness of horocycle foliations. J. Differ. Geom. 10, 225-238 (1975)

13. Margulis, G.A.: Certain measures associated with $U$-flows on compact manifolds. Funct. Anal. Appl. 4, 55-67 (1970)

14. Parry, W., Pollicott, M.: An analogue of the prime number theorem for closed orbits of Axiom $A$ flows. Ann. Math. 118, 573-591 (1983)

15. Plante, J.F.: Anosov flows. Am. J. Math. 94, 729-754 (1972)

16. Pollicott, M.: On the rate of mixing of Axiom $A$ flows. Invent. Math. 81, 413-426 (1985)

17. Pollicott, M.: Meromorphic extensions of generalised zeta functions. Invent. Math. 85, 147-164 (1986)

18. Ratner, M.E.: Markovian partitions for $Y$-flows on three-dimensional manifolds. Mat. Zumetki 6 (6), 693-704 (1969)

19. Ratner, M.E.: Markov partitions for Anosov flows on $n$-dimensional manifolds. Isr. J. Math. 15, 102-114 (1973)

20. Ratner, M.E.: The rate of mixing for geodesic and horocycle flows. Erg. Th. \& Dynam. Syst. 7, 267-288 (1987)

21. Ruelle, D.: Resonances for Axiom $A$ flows J. Differ. Geom.

22. Ruelle, D.: Extension of the concept of Gibbs state in one dimension and an application to resonances for Axiom $A$ diffeomorphisms J. Differ. Geom.

23. Ruelle, D.: Thermodynamic formalism. New York: Addison-Wesley 1978

24. Ruelle, D.: Zeta functions for expanding maps and Anosov flows. Invent. Math. 34, 231-242 (1976)

25. Ruelle, D., Sullivan, D.: Currents flows and diffeomorphisms. Topology 14, 319-327 (1975)

26. Schwartzman, S.: Asymptotic cycles. Ann. Math. 66, 270-284 (1957)

27. Smale, S.: Differentiable dynamical systems. Bull. Am. Math. Soc. 73, 747-817 (1967)

28. Sullivan, D.: Cycles for the dynamical study of foliated manifolds and complex manifolds. Invent. Math. 36, 225-255 (1976)

Communicated by J.-P. Eckmann

Received December 1, 1986; in revised form May 13, 1987 